\title{
Multilateration Localization Based on Singular Value Decomposition for 3D Indoor Positioning
}

\author{
Jihoon Yang, Haeyoung Lee and Klaus Moessner \\ Institute for Communication Systems (ICS) \\ Department of Eletrical and Electronic Engineering \\ University of Surrey \\ Guildford, Surrey, GU2 7XH, United Kingdom \\ Email: \{j.yang, haeyoung.lee, k.moessner\}@ surrey.ac.uk
}

\begin{abstract}
Localization is crucial for various applications, this includes resource coordination in small and ultra-small cells, as well as the whole range of Location Based Service (LBS). Multilateration is a localization technique that is based on distance measurements between multiple reference nodes and a target node. This paper introduces a multilateration localization approach that uses Singular Value Decomposition (SVD) for 3D indoor positioning. The paper provides a mathematical multilateration formulation which considers the coordinates of the reference nodes and the relative distance between transmitting nodes. In practical deployments, the relative distance can be estimated using RSSI; we apply Kalman filtering to the RSSI measurements aiming to get a more accurate RSSI value. The approach is complemented by using two selection methods which help chosing the best nodes for multilateration computation. The paper concludes with a discussion of the experimental evaluation results obtained.
\end{abstract}

Index Terms-Kalman Filter, Localization, Multilateration, RSSI, Sigular Value Decomposition (SVD)

\section{INTRODUCTION}

The increase in numbers of mobile computing devices and as well as almost ubiquitous availability of local-area wireless networks has increased the interest in location-based services [1]. Mass-customization as well as the ever shrinking cell sizes in current (and future networks) further increase this interest. Looking at mass-customization and large-scale individualisation, context-awareness for applications such as mobile advertising/billing, logistics, and tracking [2][3], is a must and being able to accurately locate a user or device is one of the most significant aspects of context. While location-based services are reliant on accurate position localization, energy efficient and inexpensive localization is a must for applications operating on battery-constrained mobile devices [4].

Outdoors, a wide range of location-based services are based on satelitte based systems like the global positioning system (GPS), or on cell-based localisation. However, for an indoor environment such as hospitals, airports, and shopping malls, localization cannot be carried out effectively by GPS [5] since the signal from satellites needs line of sight and is typically too much attenuated, e.g., windows, walls, furniture, or due to the mobility of people, and some interference and noise from other wired and wireless networks [6]. Thus, indoor localization needs other solutions [7][8].
There is much research into indoor localization. In most approaches, the indoor positioning process is divided into two phases: signal measurement and location calculation. In the first phase, signals are transmitted between nodes and the signal properties are extracted by using methods such as TOA (Time of Arrival) [9], TDOA (Time Difference of Arrival) [10], AOA (Angle of Arrival) [11] or RSSI (Received Signal Strength Indicator). TOA, TDOA and AOA-based solutions are generally more energy dependent and/or require dedicated hardware. Pure RSSI-based approaches, in general, are simple and low cost with no extra hardware cost [12]. Thus, in this paper, RSSI-based localization is considered for a practical indoor localization solution. In the second phase, the physical coordinates of the target node are calculated based on the signal parameters measured in the first phase, using the known coordinates of the reference nodes as anchor points. Typically triangulation and trilateration techniques are used for this. While triangulation is based on the measurement of angles for AOA [13], trilateration makes use of the estimated distances between the target node and three known reference nodes, which should be non-co-linear [14]. Trilateration can be extended into multilateration by using multiple (i.e., more than three) reference nodes to estimate the coordinates of a target node. Recent studies in [15][16][17][18][19] have proposed multilateration algorithms but only for target location in 2D coordinate systems, i.e. $(x, y)$.

In this paper, we formulate the multilateration problem via Singular Value Decomposition (SVD) in order to estimate the location of target nodes in three-dimensional (3D) environments. For given multiple reference nodes, distance between reference nodes and the target node is estimated using RSSI, and then the location of the target node, i.e., the 3D coordinate $(x, y, z)$, can be calculated. Typically, RSSI provides rather inaccurate information; in order to reduce RSSI fluctuation that leads to inaccurate distance estimation, we apply a Kalman filter [20] to the RSSI measurements. In addition, the selection of the reference nodes that are used for the RSSI estimation is evaluated.

The remainder of this paper is organized as follows: Section II presents the RRSI model and Kalman filtering applied on the RSSI measurements. The details of the multilateration problem are explained in Section III. After describing the experimental 
set up and analysing the results in Section IV, our conclusions are provided in Section V.

\section{RSSI BASED KALMAN FILTER}

The Received Signal Strength Indicator (RSSI) value is associated with the power of a received radio signal (measured in $\mathrm{dBm}$ ), and represents the relationship between a transmission and a received power. Thus it is used for relative distance measurements between transmitting and receiving nodes.

\section{A. RSSI Model}

The relationship between RSSI and distance can be determined according to the Friis transmission equation [21].

$$
P_{r}(d)=\frac{P_{t} G_{t} G_{r} \lambda^{2}}{4 \pi^{2} d^{2} L}
$$

where $P_{r}$ is the received power of a transmitted signal and $P_{t}$ is the transmission power. $G_{t}$ and $G_{r}$ denote the antenna gain of the transmitter and receiver, respectively. $d$ is the distance between transmitter and receiver, and $L$ is the system loss factor. $\lambda$ denotes the wavelength.

RSSI is measured in $d B m$ and the distance model is estimated with the Log-Distance pathloss model [22]:

$$
P_{r}(d)[d B m]=P_{0}\left(d_{0}\right)[d B m]-10 \eta \log _{10}\left(\frac{d}{d_{0}}\right)-\chi_{\sigma},
$$

where $P_{0}\left(d_{0}\right)$ is the reference power value in $d B m$ at a reference distance from the transmitter. $\eta$ is the signal propagation constant depending on the environment of propagation, typically between 2 to $4 . \chi_{\sigma}$ is a zero mean Gaussian distributed random variable with standard deviation $\sigma$ which accounts for the random effects of shadowing and for channel model inaccuracy. For a given $P_{r}(d)$, the relative distance between a transmitter and receiver can be calculated by

$$
d=d_{0} \cdot 10 \frac{\frac{-P_{r}(d)[d B m]+P_{0}\left(d_{0}\right)[d B m]-\chi_{\sigma}}{10 \eta}}{10} .
$$

Fig. 1a shows the relation between the RSSI and distance. The received signal strength is clearly influenced by distance but the amount of noise is substantial. The calculated distance using (3) is shown in Fig. 1b. Fig. 1 shows the estimated distance are roughly correct with a lot of noise while RSSI values are fluctuatingg widely. RSSI values are heavily influenced by environment, movement of humans inside the building, reflections such as walls and furniture, and interference of other signals. Without loss of generality, these cause noise on the RSSI measurement. Therefore we need to remove noise elements in order to calculate more accurate distances from RSSI values.

\section{B. Kalman Filter for RSSI Measurement}

The Kalman filter is one of the popular data fusion algorithms in the field of signal processing, and can estimate unobserved variables based on noisy measurements. The Kalman filter model is based on a state-space approach of linear dynamic systems in the time domain, and assumes that the

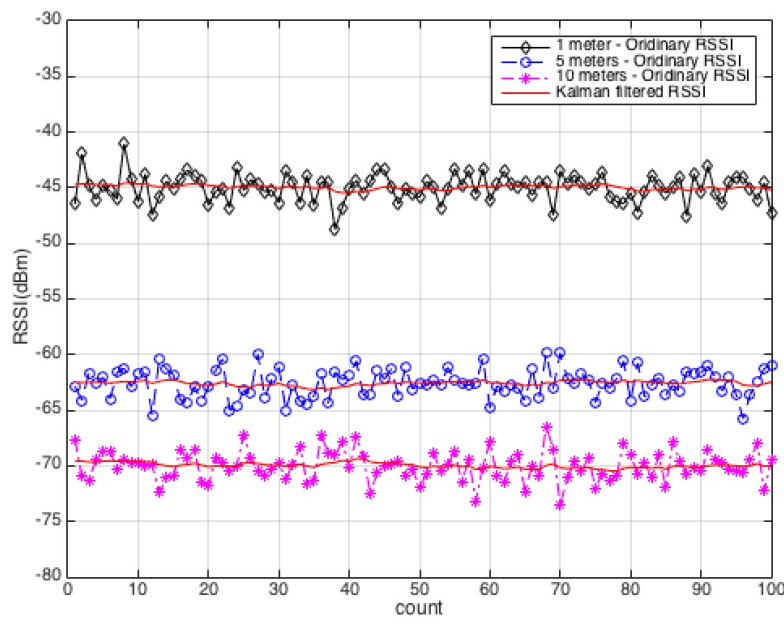

(a) RSSI measurements at different diatances

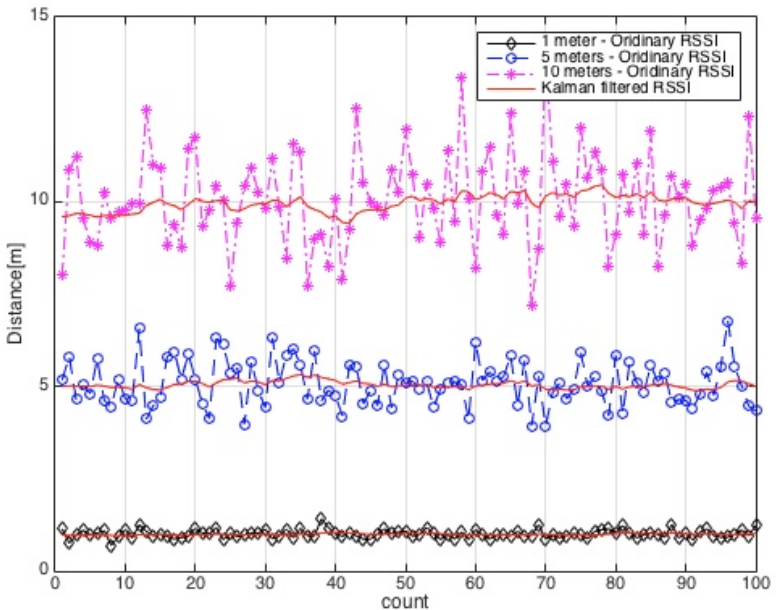

(b) Calculated distance from measured RSSI using the pathloss model

Fig. 1: RSSI behaviour

state at a time $t$ is evolved from the prior state at time $t-1$ according to the state-space equation:

$$
\mathbf{x}_{t}=\mathbf{F}_{t} \mathbf{x}_{t-1}+\mathbf{B}_{\mathbf{t}} \mathbf{u}_{t}+\mathbf{w}_{t}
$$

where $\mathbf{x}_{t}$ is the state vector at time $t, \mathbf{u}_{t}$ is the control the input vector, $\mathbf{F}_{t}$ is the state transition matrix which applies the effect of each state at time $t-1$ on the system state at time $t, \mathbf{B}_{t}$ is the control input matrix which applies the effect of each control input vector $\mathbf{u}_{t}$, and $\mathbf{w}_{t}$ is the vector containing the process noise which is assumed to be zero mean multivariate normal distribution with covariance $\mathbf{Q}_{t}$. And the system's measurements can be performed as:

$$
\mathbf{z}_{t}=\mathbf{H}_{t} \mathbf{x}_{t}+\mathbf{v}_{t}
$$

where $\mathbf{z}_{t}$ is the measurement (or observation) vector at time $t$, $\mathbf{H}_{t}$ is the transformation matrix which maps the state vector into the measurement space, and $\mathbf{v}_{t}$ is the vector containing the measurement noise which is assumed to be zero mean Gaussian white noise with covariance $\mathbf{R}_{t}$. 
The Kalman filter algorithm has two distinct sets of equations as time update (prediction) and measurement update (correction). The prediction equations of the a priori state estimate $\hat{\mathbf{x}}_{t \mid t-1}$ and the a prior estimate covariance matrix $\mathbf{P}_{t \mid t-1}$ are:

$$
\begin{aligned}
\hat{\mathbf{x}}_{t \mid t-1} & =\mathbf{F}_{t} \hat{\mathbf{x}}_{t-1 \mid t-1}+\mathbf{B}_{t} \mathbf{u}_{t} \\
\mathbf{P}_{t \mid t-1} & =\mathbf{F}_{t} \mathbf{P}_{t-1 \mid t-1} \mathbf{F}_{t}^{T}+\mathbf{Q}_{t}
\end{aligned}
$$

where $\hat{\mathbf{x}}_{t-1 \mid t-1}$ is the a posteriori state estimate at time $t-1$, $\mathbf{P}_{t-1 \mid t-1}$ is the a posteriori error covariance matrix at time $t-1$.

The measurement update equations of the a posteriori state estimate $\hat{\mathbf{x}}_{t \mid t}$ and the a posteriori estimate covariance matrix $\mathbf{P}_{t \mid t}$ are given by

$$
\begin{gathered}
\hat{\mathbf{x}}_{t \mid t}=\hat{\mathbf{x}}_{t \mid t-1}+\mathbf{K}_{t}\left(\mathbf{z}_{t}-\mathbf{H}_{t} \hat{\mathbf{x}}_{t \mid t-1}\right) \\
\mathbf{P}_{t \mid t}=\mathbf{P}_{t \mid t-1}-\mathbf{K}_{t} \mathbf{H}_{t} \mathbf{P}_{t \mid t-1}
\end{gathered}
$$

where the Kalman gain $\mathbf{K}_{t}$ can be derived as

$$
\mathbf{K}_{t}=\mathbf{P}_{t \mid t-1} \mathbf{H}_{t}^{T}\left(\mathbf{H}_{t} \mathbf{P}_{t \mid t-1} \mathbf{H}_{t}^{T}+\mathbf{R}_{t}\right)^{-1} .
$$

For RSSI measurements: as the signal of RSSI is a scalar value and there is no control input $\mathbf{u}_{t}$, the state transition matrix is 1 , i.e. $\mathbf{F}_{t}=1, \mathbf{u}_{t}=0$. Also the measurement value of RSSI is composed of the state value and noise, so the transformation matrix is just 1 , i.e. $\mathbf{H}_{t}=1$. To apply Kalman filtering to RSSI measurements, we assume that a wireless device does not move and its position is fixed. In other words, it expects to get a constant RSSI signal, everything else contains noise. Thus the state-space equations of (4) and (5) can be simple as following:

$$
\begin{gathered}
\mathbf{x}_{t}=\mathbf{x}_{t-1}+\mathbf{w}_{t} \\
\mathbf{z}_{t}=\mathbf{x}_{t}+\mathbf{v}_{t}
\end{gathered}
$$

And also, (6)-(10) can be arranged to (13)-(17), respectively. Time update (prediction):

$$
\begin{gathered}
\hat{\mathbf{x}}_{t \mid t-1}=\hat{\mathbf{x}}_{t-1 \mid t-1} . \\
\mathbf{P}_{t \mid t-1}=\mathbf{P}_{t-1 \mid t-1}+\mathbf{Q}_{t} .
\end{gathered}
$$

Measurement update (correction):

$$
\begin{gathered}
\hat{\mathbf{x}}_{t \mid t}=\hat{\mathbf{x}}_{t \mid t-1}+\mathbf{K}_{t}\left(\mathbf{z}_{t}-\hat{\mathbf{x}}_{t \mid t-1}\right) . \\
\mathbf{P}_{t \mid t}=\left(1-\mathbf{K}_{t}\right) \mathbf{P}_{t \mid t-1}
\end{gathered}
$$

where $\mathbf{K}_{t}$ becomes

$$
\mathbf{K}_{t}=\frac{\mathbf{P}_{t \mid t-1}}{\mathbf{P}_{t \mid t-1}+\mathbf{R}_{t}}
$$

We have applied the Kalman filter algorithm, (13)-(17), to the RSSI data to remove noise, and then the effect of the Kalman filter on ordinary RSSI data sampled is shown in Fig. 1. The Kalman filter can remove a large part of the noise from the ordinary RSSI. So the Kalman filter makes optimal use of imprecise RSSI data in this paper.

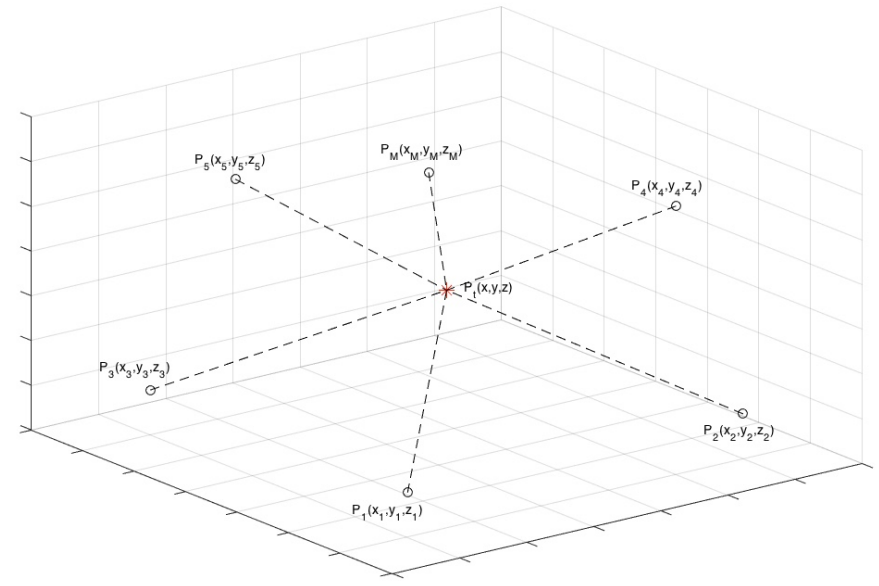

Fig. 2: Multilateration in three dimensions

\section{Multilateration-BASEd Localization}

In this section, we presents a mathematical multilateration model and propose a solution method based on singular value decomposition.

\section{A. Multilateration Model}

$M$ reference nodes are given by their 3D coordinates, i.e., $P_{i}\left(x_{i}, y_{1}, z_{i}\right)$ where $i \in M$. The relative distances $d_{i}$ between the reference nodes $i$ and the target node $P(x, y, z)$ are measured by using RSSI. Determination of the target coordinate $(x, y, z)$ is equivalent to finding the solution of the following equations.

$$
\begin{gathered}
\left(x-x_{1}\right)^{2}+\left(y-y_{1}\right)^{2}+\left(z-z_{1}\right)^{2}=d_{1}{ }^{2} \\
\left(x-x_{2}\right)^{2}+\left(y-y_{2}\right)^{2}+\left(z-z_{2}\right)^{2}=d_{2}{ }^{2} \\
\vdots \\
\left(x-x_{M}\right)^{2}+\left(y-y_{M}\right)^{2}+\left(z-z_{M}\right)^{2}=d_{M}{ }^{2} .
\end{gathered}
$$

(18) can be arranged as following:

$$
\begin{gathered}
x^{2}+y^{2}+z^{2}-2 x_{1} x-2 y_{1} y-2 z_{1} z \\
=d_{1}{ }^{2}-x_{1}{ }^{2}-y_{1}{ }^{2}-z_{1}{ }^{2} \\
x^{2}+y^{2}+z^{2}-2 x_{2} x-2 y_{2} y-2 z_{2} z \\
=d_{2}{ }^{2}-x_{2}{ }^{2}-y_{2}{ }^{2}-z_{2}{ }^{2} \\
\vdots \\
x^{2}+y^{2}+z^{2}-2 x_{M} x-2 y_{M} y-2 z_{M} z \\
=d_{M}{ }^{2}-x_{M}{ }^{2}-y_{M}{ }^{2}-z_{M}{ }^{2} .
\end{gathered}
$$

(19) can be represented in a form of matrix.

$$
\mathbf{A x}=\mathbf{b}
$$


where $\mathbf{A}$ is a $M \times N$ matrix and $\mathbf{x}$ is a $N \times 1$ matrix. $\mathbf{b}$ is a $M \times 1$ matrix such as

$$
\begin{aligned}
\mathbf{A} & =\left[\begin{array}{cccc}
1 & -2 x_{1} & 2 y_{1} & 2 z_{1} \\
1 & -2 x_{2} & 2 y_{2} & 2 z_{2} \\
\vdots & \vdots & \vdots & \vdots \\
1 & -2 x_{M} & 2 y_{M} & 2 z_{M}
\end{array}\right], \mathbf{x}=\left[\begin{array}{c}
x^{2}+y^{2}+x^{2} \\
x \\
y \\
z
\end{array}\right], \\
\text { and } \mathbf{b} & =\left[\begin{array}{c}
d_{1}{ }^{2}-x_{1}{ }^{2}-y_{1}{ }^{2}-z_{1}{ }^{2} \\
d_{2}{ }^{2}-x_{2}{ }^{2}-y_{2}{ }^{2}-z_{2}{ }^{2} \\
\vdots \\
d_{M}{ }^{2}-x_{m}{ }^{2}-y_{M}{ }^{2}-z_{M}{ }^{2}
\end{array}\right] .
\end{aligned}
$$

It should be noted that all elements in matrix $\mathbf{A}$ are composed of the known coordinates of the reference nodes and $\mathbf{b}$ is comprised of distances between the target node and all reference nodes. Thus, for allgiven coordinates of reference nodes and each measured distance between nodes, the 3D coordinate $(x, y, z)$ of the target node can be obtained by solving (20). In other words, the multilateration problem can be converted to a linear algebra method.

If $\mathbf{A}$ in (20) is invertible and has a full rank, the only one solution of $\mathbf{A x}=\mathbf{b}$ is $\mathbf{x}=\mathbf{A}^{-1} \mathbf{b}$. Otherwise, the unique solution can not be determined from $\mathbf{x}=\mathbf{A}^{-1} \mathbf{b}$. While $M$ of $\mathbf{A}$ and $\mathbf{b}$ is decided by the number of reference nodes, $N$ of $\mathbf{A}$ and $\mathbf{x}$ is determined by the target position's dimension, e.g., $2 \mathrm{D}$ or $3 \mathrm{D}$.

Case 1: 2D localization

It means $z_{M}=0$ and $z=0$. Thus $\mathbf{A}$ in (20) is the $M \times 3$ matrix, $\mathbf{x}$ is the $3 \times 1$ matrix, and $\mathbf{b}$ is the $M \times 1$ matrix.

$M=3$ (Trilateration): $\mathbf{A}$ is a square matrix, but the unique solution exists if and only if $\operatorname{rank}(A)=M$.

$M>3$ (Multilateration): $\mathbf{A}$ is a rectangular matrix, and is not invertible.

Case 2: 3D localization

A in (20) is a $M \times 4$ matrix, $\mathbf{x}$ is a $4 \times 1$ matrix, and $\mathbf{b}$ is a $M \times 1$ matrix.

$M=3$ (Trilateration): $\mathbf{A}$ is a rectangular matrix, and is not invertible. A unique solution does not exist.

$M=4$ (Multilateration): $\mathbf{A}$ is a square matrix, but a unique solution exists if and only if $\operatorname{rank}(A)=M$.

$M>4$ (Multilateration): $\mathbf{A}$ is a rectangular matrix, and is not invertible.

Therefore we need to find the unique solution $\mathrm{x}$ based on singular value decomposition for all above cases.

\section{B. Solution of Multilateration}

In general, solution that has the minimum square norm (i.e., the closest point to the origin) can be selected. It can be stated as follows.

$$
\begin{array}{ll}
\operatorname{minimize} & \mathbf{x}^{T} \mathbf{x} \\
\text { subject to } & \mathbf{A x}=\mathbf{b}
\end{array}
$$

If $\mathbf{A}$ in (20) is a $M \times N$ matrix with singular values $\left\{\sigma_{1}, \sigma_{2}, \ldots, \sigma_{N}\right\}, \mathbf{A}$ can be decomposed by Singular Value
Decomposition (SVD).

$$
\mathbf{A}=\mathbf{U} \mathbf{\Sigma} \mathbf{V}^{T}
$$

where $\mathbf{U}=\left[\mathbf{u}_{1}, \mathbf{u}_{2}, \ldots, \mathbf{u}_{M}\right]$ is a $M \times M$ orthogonal matrix. $\boldsymbol{\Sigma}$ is a $M \times N$ diagonal matrix and $\mathbf{V}=\left[\mathbf{v}_{1}, \mathbf{v}_{2}, \ldots, \mathbf{v}_{N}\right]$ is a $N \times N$ orthogonal matrix. The columns $\mathbf{u}_{i}(i=1,2, \ldots, M)$ of $\mathbf{U}$ are called left singular vectors of $\mathbf{A}$, and the columns $\mathbf{v}_{i}$ $(i=1,2, \ldots, N)$ are called right singular vectors of $\mathbf{A}$. The singular values of $\mathbf{A}$ are $\sigma_{1} \geq \sigma_{2} \geq \ldots \geq \sigma_{N}>0$, then $\boldsymbol{\Sigma}$ has the block form as follows.

$$
\boldsymbol{\Sigma}=\left[\begin{array}{cccc}
\sigma_{1} & 0 & \cdots & 0 \\
0 & \sigma_{2} & \cdots & 0 \\
\vdots & \vdots & \ddots & \vdots \\
0 & 0 & \cdots & \sigma_{N} \\
0 & 0 & 0 & 0 \\
\vdots & \vdots & \vdots & \vdots \\
0 & 0 & 0 & 0
\end{array}\right]=\left[\begin{array}{c}
\boldsymbol{\Sigma}_{N} \\
0
\end{array}\right]
$$

In order to solve (22), we use the following.

$$
\begin{aligned}
\mathbf{A A}^{T} & =\mathbf{U}\left[\begin{array}{c}
\boldsymbol{\Sigma}_{N} \\
0
\end{array}\right] \mathbf{V}^{T} \mathbf{V}\left[\begin{array}{ll}
\boldsymbol{\Sigma}_{N} & 0
\end{array}\right] \mathbf{U}^{T} \\
& =\mathbf{U}\left[\begin{array}{cc}
\boldsymbol{\Sigma}_{N}^{2} & 0 \\
0 & 0
\end{array}\right] \mathbf{U}^{T},
\end{aligned}
$$

where $\boldsymbol{\Sigma}_{N}^{2}$ is a $N \times N$ diagonal matrix with $\sigma_{i}^{2}$ on the $i$ th diagonal. Since both $\boldsymbol{\Sigma}_{n}$ and $\boldsymbol{\Sigma}_{N}^{2}$ have no zero elements on the diagonal, $\mathbf{A} \mathbf{A}^{T}$ is an invertible, symmetric, and positivedefinite matrix.

We now solve the problem stated in (22) using Lagrange multipliers $\lambda$. Let

$$
H=\mathbf{x}^{T} \mathbf{x}+\lambda^{T}(\mathbf{A} \mathbf{x}-\mathbf{b}) .
$$

The solution is found by solving the equation $\partial H / \partial \mathbf{x}$ and then ensuring that the constraint $\mathbf{A x}=\mathbf{b}$ holds. First solve for $\mathbf{x}$ :

$$
\begin{aligned}
\frac{\partial H}{\partial \mathbf{x}} & =0 . \\
2 \mathbf{x}^{T}+\lambda^{T} \mathbf{A} & =0 . \\
\mathbf{x} & =-\frac{1}{2} \mathbf{A}^{T} \lambda .
\end{aligned}
$$

Using the fact that $\mathbf{A} \mathbf{A}^{T}$ is invertible, choose $\lambda$ to ensure that

$$
\begin{aligned}
\mathbf{A x} & =\mathbf{b} . \\
\mathbf{A}\left(-\frac{1}{2} \mathbf{A}^{T} \lambda\right) & =\mathbf{b} . \\
\lambda & =-2\left(\mathbf{A A}^{T}\right)^{-1} \mathbf{b} .
\end{aligned}
$$

Substitute (28) into (27) to get an expression of $\mathbf{x}$.

$$
\begin{aligned}
\mathbf{x} & =\mathbf{A}^{T}\left(\mathbf{A} \mathbf{A}^{T}\right)^{-1} \mathbf{b} \\
& =\mathbf{V}\left[\begin{array}{ll}
\boldsymbol{\Sigma}_{N} & 0
\end{array}\right] \mathbf{U}^{T}\left(\mathbf{U}\left[\begin{array}{cc}
\boldsymbol{\Sigma}_{N}^{2} & 0 \\
0 & 0
\end{array}\right] \mathbf{U}^{T}\right)^{-1} \mathbf{b} \\
& =\mathbf{V}\left[\begin{array}{ll}
\boldsymbol{\Sigma}_{N} & 0
\end{array}\right] \mathbf{U}^{T} \mathbf{U}\left[\begin{array}{cc}
\boldsymbol{\Sigma}_{N}^{-2} & 0 \\
0 & 0
\end{array}\right] \mathbf{U}^{T} \mathbf{b} \\
& =\mathbf{V}\left[\begin{array}{ll}
\boldsymbol{\Sigma}_{N}^{-1} & 0
\end{array}\right] \mathbf{U}^{T} \mathbf{b} .
\end{aligned}
$$


The pseudoinverse of $\mathbf{A}, \mathbf{A}^{+}(N \times M)$ is defined by

$$
\mathbf{A}^{+}=\mathbf{V} \Sigma^{+} \mathbf{U}^{T}
$$

where $\boldsymbol{\Sigma}^{+}$is a $N \times M$ matrix as follows.

$$
\boldsymbol{\Sigma}^{+}=\left[\begin{array}{ccccccc}
\frac{1}{\sigma_{1}} & 0 & \ldots & 0 & 0 & \ldots & 0 \\
0 & \frac{1}{\sigma_{2}} & \cdots & 0 & 0 & \ldots & 0 \\
\vdots & \vdots & \ddots & \vdots & \vdots & \ldots & \vdots \\
0 & 0 & \cdots & \frac{1}{\sigma_{N}} & 0 & \ldots & 0
\end{array}\right]
$$

Finally, we can write the solution of (20) as

$$
\mathbf{x}=\mathbf{A}^{+} \mathbf{b}=\mathbf{V} \Sigma^{+} \mathbf{U}^{T} \mathbf{b} .
$$

The 3D coordinates $(x, y, z)$ of the target node can be obtained by solving (32).

\section{Selection of Participating Nodes}

For a given set of available reference nodes, certain participating nodes need to be chosen in order to apply the multilateration localization approach. In order to investigate the impact of a participating nodes' selection mechanism, we consider the two following cases.

1) Case 1: Selection of reference nodes having stable RSSI: In this approach, reference nodes having less fluctuated RSS are selected. As an indicator of RSSI fluctuation, the standard deviation is identified. First, the average RSSI value of each reference node, $\gamma_{i}$ is calculated as following:

$$
\gamma_{i}=\frac{\sum_{k=1}^{C} P r_{k}^{i}}{C}
$$

where $\operatorname{Pr}_{k}^{i}$ is RSSI value received from $i$ th reference node at $k$ times. Then, the standard deviation (s.d) is derived as following.

$$
\xi_{i}=\left[\frac{\sum_{k=1}^{C}\left(P r_{k}^{i}-\gamma_{i}\right)^{2}}{C-1}\right]^{\frac{1}{2}}
$$

where $\xi_{i}$ indicates the standard deviation of RSSI value received from $i$ th reference node. Let $\Xi=\left\{\xi_{1}, \xi_{2}, \cdots, \xi_{M}\right\}$. We can sort $\Xi$ in ascending order according to the standard deviation, $\xi_{i}$, where $i \in\{1,2, \cdots, M\}$. Then, the set of sorted standard deviation of RSSI values from each reference node can be expressed as

$$
\hat{\Xi}=\left\{\cdots, \xi_{i}, \xi_{j}, \cdots\right\}
$$

where $\xi_{i} \leq \xi_{j}$ and $1 \leq i, j \leq M$. When $N_{c}$ number of reference nodes are required, participating nodes can be selected as nodes showing less fluctuated RSSI from $\hat{\Xi}$.
2) Case 2: Selection of reference nodes having strong RSSI: In this approach, reference nodes having strong RSS are selected. First, the average RSSI value of each reference node, $\gamma_{i}$ is calculated as following:

Let $\gamma_{i}^{t}$ be the RSSI value from $i^{t h}$ reference node at time $t$ where $i \in\{1,2, \cdots, M\}$. Then, the set of RSSI value can be expressed as

$$
\Gamma^{t}=\left\{\gamma_{1}^{t}, \gamma_{2}^{t}, \cdots, \gamma_{M}^{t}\right\}
$$

$\Gamma^{t}$ can be sorted in descending order with the RSSI value from each reference node, $\gamma_{i}^{t}$. Then,

$$
\hat{\Gamma}^{t}=\left\{\cdots, \gamma_{i}^{t}, \gamma_{j}^{t}, \cdots\right\}
$$

where $\gamma_{i}^{t} \geq \gamma_{j}^{t}$ and $1 \leq i, j \leq M, \hat{\Gamma}^{t}(j)$ the RSSI value which is ranked $j$ th.

\section{EXPERIMENT}

In our experiments we use TelosB motes and XM100 motes using the CC2420 radio chip as shown in 3a. They are IEEE 802.15.4 compliant, and the $\mathrm{CC} 2420$ operates in $2.4 G H z$ to $2.4835 \mathrm{GHz}$ (ISM band) with a data rate of $256 \mathrm{kbps}$.

Since the experiments' aim is the 3D position estimation of a target node, we do not collect any sensor information other than RSSI. All measurements are performed in a seminar room (of the size $7 m \times 16 m \times 3 m$ ) in the ICS (5GIC) building at University of Surrey as shown in Fig. 3.

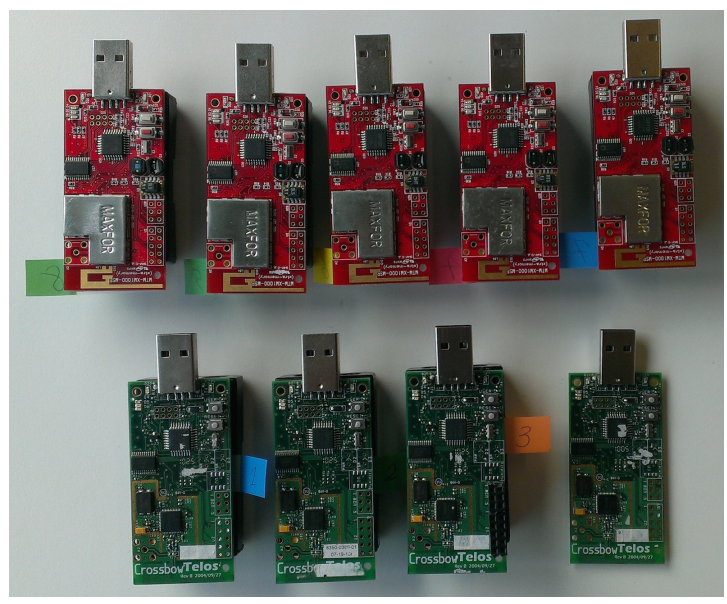

(a) TelosB motes and XM1000 motes

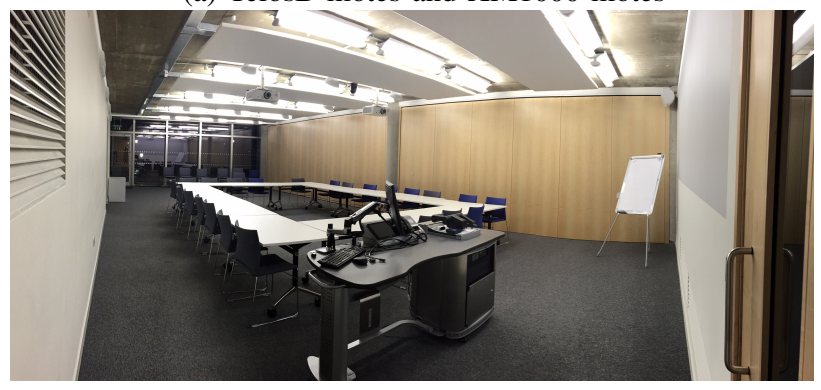

(b) the Seminar room in ICS building at University of Surrey

Fig. 3: Experiment Environment 
TABLE I: 3D coordinates of reference nodes

\begin{tabular}{|c||c|c|c|}
\hline \multicolumn{1}{|c||}{\multirow{2}{*}{$\begin{array}{l}\text { Reference } \\
\text { node }\end{array}$}} & \multicolumn{3}{c|}{ coordinates } \\
\cline { 2 - 4 } & $\mathrm{x}[\mathrm{m}]$ & $\mathrm{y}[\mathrm{m}]$ & $\mathrm{z}[\mathrm{m}]$ \\
\hline$P_{1}$ & 0 & 5.0 & 0.0 \\
\hline$P_{2}$ & 0 & 0 & 2.4 \\
\hline$P_{3}$ & 7.0 & 4.0 & 0.9 \\
\hline$P_{4}$ & 3.0 & 11.5 & 0.7 \\
\hline$P_{5}$ & 1.0 & 15.0 & 1.7 \\
\hline$P_{6}$ & 5.5 & 13.0 & 0.8 \\
\hline$P_{7}$ & 2.5 & 3.5 & 0.5 \\
\hline$P_{8}$ & 4.5 & 9.5 & 2.5 \\
\hline
\end{tabular}

In this experiment we have defined performance metrics such as Root Mean Square Error (RMSE) of the estimated 3D position as following:

$$
R M S E=\frac{\sum_{k=1}^{C} \sqrt{(x-\bar{x})^{2}+(y-\bar{y})^{2}+(z-\bar{z})^{2}}}{C}
$$

where $(x, y, z)$ is the actual point while the $(\bar{x}, \bar{y}, \bar{z})$ is the average estimated point.

The experiment setup consists of eight reference nodes and one target node. The 3D coordinates of the reference nodes which have been deployed in the experiment area are listed in Table I. The target node is located at $P=(5.0,8.0,0.7)$ meters.

First, the RSSI values are measured and the Kalman filtering algorithm in (13)-(17) is applied. Then, the 3D coordinates of the target node are calculated using (32). It should be noted that the covariance values of (14) and (17) are fixed such as $\mathbf{Q}_{t}=1$ and $\mathbf{R}_{t}=100$ in this experiment, respectively.

We performed 100 trials to estimate the 3D coordinates of the target node using the above method. The estimated positions in association with different numbers of participating reference nodes are shown in Fig. 4 and 5.

The table II shows the experimental results for different numbers of anchor nodes. The anchor nodes in the multilateration computation are chosen using (35) and (37), respectively. Fig. 6 depicts the RMSE. It can be seen that increasing the number of reference nodes used in the computation of multilateration localization generates better results, when using up to 6 nodes. However the estimation error increases when more nodes (i.e., 7th and 8th nodes) are added to the multilateration localization computation. This is different from the theoretical assumption that an increase in number of nodes results in higher location accuracy. In addition, the same phenomenon occurs for two selection cases described in subsection IIIC. Based on the results, it can be seen that higher numbers of nodes in a specified size space can cause interference among transmitting nodes. This can ultimately impact location accuracy. The impact of the participating nodes' selection mechanism is not significant on the performance of the proposed multilateration localization for 3D indoor positioning.

There are some limitations to our experimental setup, the number of nodes and the space are within the bouindaries of the room size. Therefore, the RSSI data could only be measured in a rather confined space. It is therefore necessary to verify the proposed multilateration method with respect to a large number of nodes and a variety of environments in future research. We also need to further investigate how to calculate the optimal number of participating reference nodes for multilateration localization in accordance with the indoor environmental change.

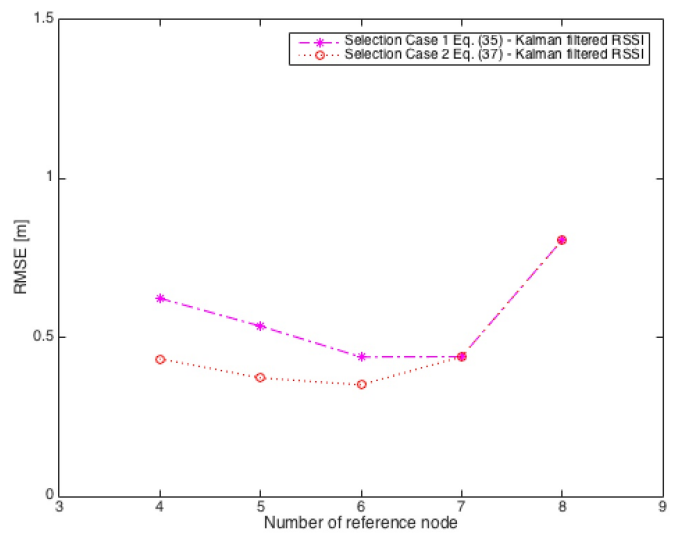

Fig. 6: RMSE of estimated locations according to the number of reference nodes

\section{CONClusion}

In this paper, we presented multilateration localization using singular value decomposition for 3D indoor positioning. We showed the mathematical multilateration formulation to be converted to a linear algebra approach. The parameters of the multilateration model were only related with the coordinates of reference nodes and relative distances between reference nodes and the target node. The distances between reference nodes and target node were estimated using RSSI, and then Kalman filtering was applied to the RSSI measurements in order to get a more accurate RSSI value. In addition, we suggested two methods which may be helpful to choose the participated nodes for multilateration computation. We evaluated the proposed method in 3D indoor positioning through the real experiment environment, and analysed the influence of the number of reference in the proposed multilateration localization. While the evaluation shows that an increase in numbers of reference nodes will improve the localization accuracy, the experiments have shown that, in practice, the use of too many nodes for multilateration will indeed reduce the accuracy again (due to increased interference). Our experimental setup did proof the validity of our approach although further evaluations in different environments are needed to proof its generality.

\section{REFERENCES}

[1] P. Bahl and V. N. Padmanabhan, "Radar: An in-building rf-based user location and tracking system," in Proc. IEEE INFOCOM 2000, vol. 2, March 2000, pp. 775-784 vol.2.

[2] N. Patwari, J. N. Ash, S. Kyperountas, A. O. Hero, R. L. Moses, and N. S. Correal, "Locating the nodes: cooperative localization in wireless sensor networks," IEEE Signal Process. Mag., vol. 22, no. 4, pp. 54-69, July 2005. 

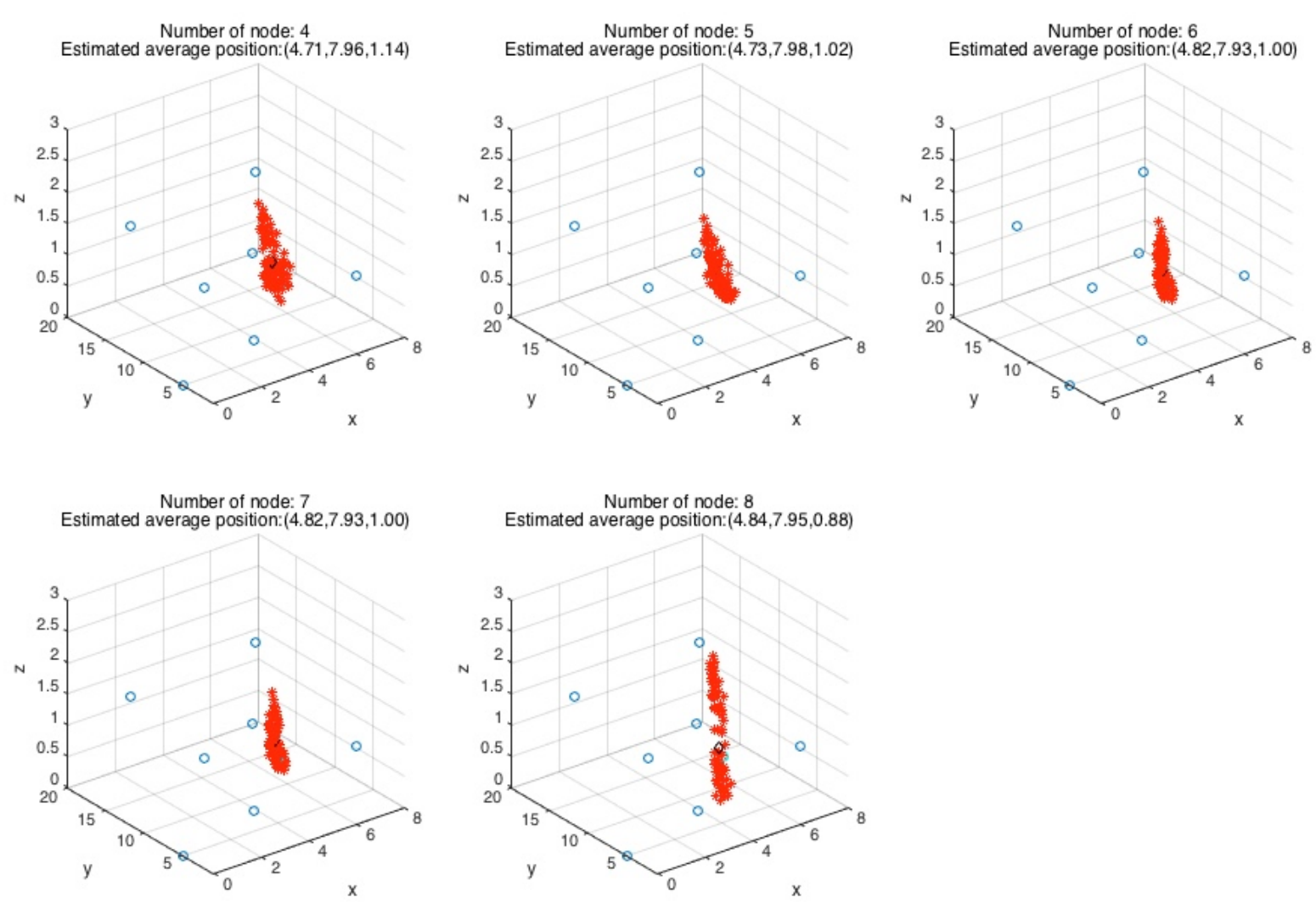

Fig. 4: Estimated 3D coordinates with various number of reference nodes chosen by (35) in multilateration computation.
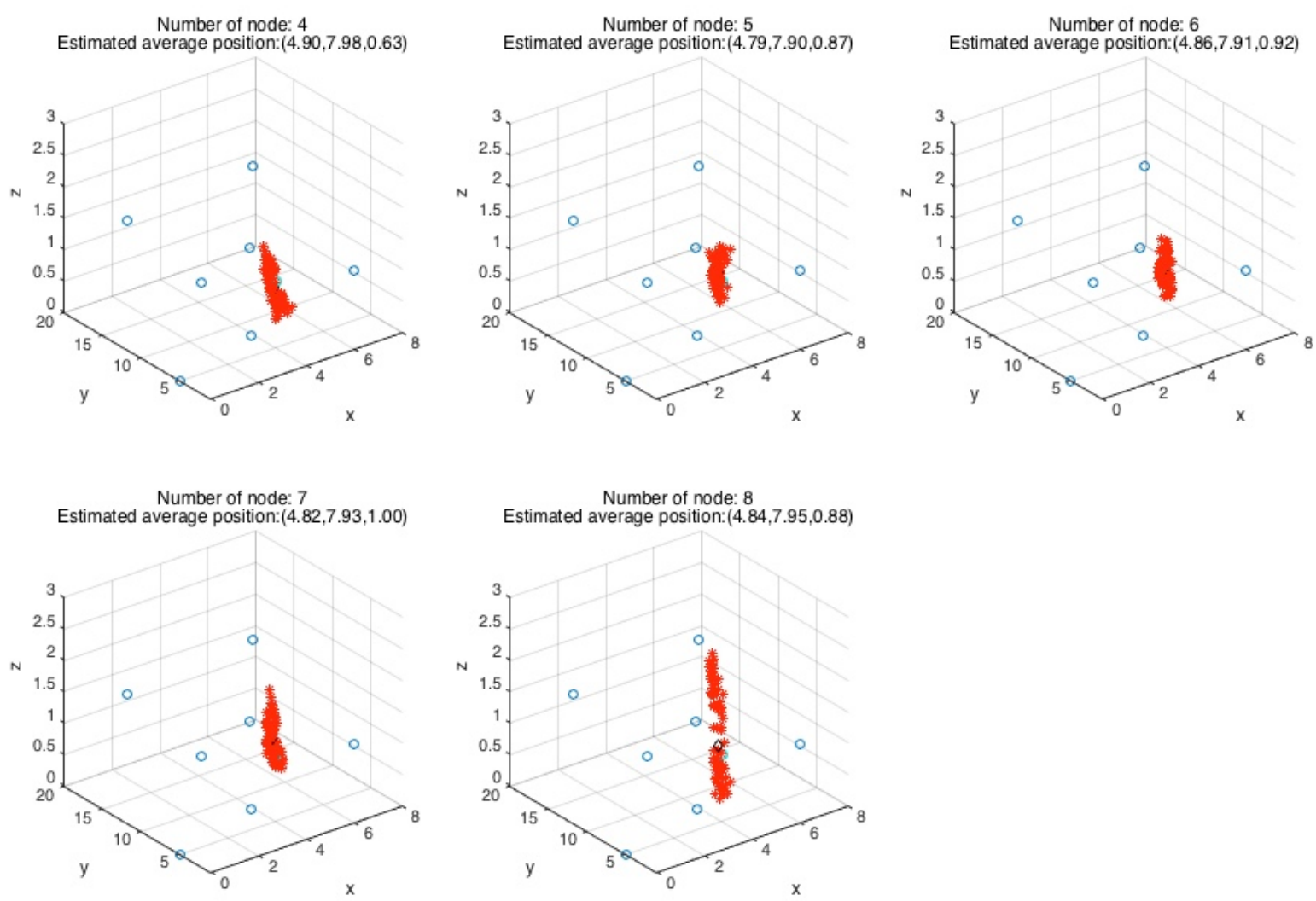

Fig. 5: Estimated 3D coordinates with various number of reference nodes chosen by (37) in multilateration computation. 
TABLE II: Experiment results corresponding to (35) and (37)

\begin{tabular}{|c||c|c|c|c||c|c|c|c|}
\hline \multicolumn{1}{|c|}{$\begin{array}{c}\text { Number } \\
\text { of nodes }\end{array}$} & \multicolumn{4}{c||}{ Selection case 1: (35) } & \multicolumn{4}{c|}{ Selection case 2: (37) } \\
\hline 4 & Estimated ave. position & RMSE & Mean & S.d. & Estimated ave. position & RMSE & Mean & S.d. \\
\hline 5 & $(4.71,7.96,1.14)$ & 0.62315 & 0.125780 & 0.35465 & $(4.90,7.98,0.63)$ & 0.43266 & 0.029601 & 0.17205 \\
\hline 6 & $(4.73,7.98,1.02)$ & 0.53565 & 0.083473 & 0.28892 & $(4.79,7.90,0.87)$ & 0.37266 & 0.019067 & 0.13808 \\
\hline 7 & $(4.82,7.93,1.00)$ & 0.43851 & 0.052147 & 0.22836 & $(4.86,7.91,0.92)$ & 0.35083 & 0.022607 & 0.15036 \\
\hline 8 & $(4.82,7.93,1.00)$ & 0.43958 & 0.049587 & 0.22268 & $(4.82,7.93,1.00)$ & 0.43958 & 0.049587 & 0.22268 \\
\hline & $(4.84,7.95,0.88)$ & 0.80661 & 0.226110 & 0.43989 & $(4.84,7.95,0.88)$ & 0.80661 & 0.193500 & 0.43989 \\
\hline
\end{tabular}

[3] A. H. Sayed, A. Tarighat, and N. Khajehnouri, "Network-based wireless location: challenges faced in developing techniques for accurate wireless location information," IEEE Signal Process. Mag., vol. 22, no. 4, pp. 24-40, July 2005.

[4] M. Ros, J. Boom, G. d. Hosson, and M. DSouza, "Indoor localisation usinga context-aware dynamic position tracking model," Int'l. J. Naviagation of Observation, pp. 1-12, 2012.

[5] Y. Gu, A. Lo, and I. Niemegeers, "A survey of indoor positioning systems for wireless personal networks," IEEE Commun. Surveys Tuts., vol. 11, no. 1, pp. 13-32, First 2009.

[6] A. M. Ladd, K. E. Bekris, A. P. Rudys, D. S. Wallach, and L. E. Kavraki, "On the feasibility of using wireless ethernet for indoor localization," IEEE Trans. Robot. Autom., vol. 20, no. 3, pp. 555-559, June 2004.

[7] Z. Farid, R. Nordin, and M. Ismail, "Recent advances in wireless indoor localization techniques and system," J. Comput. Netw. Commun., vol. 2013, pp. 1-10, 2013.

[8] D. Zhang, F. Xia, Z. Yang, L. Yao, and W. Zhao, "Localization technologies for indoor human tracking," in Proc. FutureTech'10, Busan, Korea, May 2010.

[9] B. T. Fang, "Simple solutions for hyperbolic and related position fixes," IEEE Trans. Aerosp. Electron. Syst., vol. 26, no. 5, pp. 748-753, Sep. 1990.

[10] D. J. Torrieri, "Statistical theory of passive location systems," IEEE Trans. Aerosp. Electron. Syst., vol. AES-20, no. 2, pp. 183-198, March 1984.

[11] B. D. V. Veen and K. M. Buckley, "Beamforming: a versatile approach to spatial filtering," IEEE ASSP Mag., vol. 5, no. 2, pp. 4-24, April 1988.

[12] G. Zanca, F. Zorzi, A. Zanella, and M. Zorzi, "Experimental comparison of rssi-based localization algorithms for indoor wireless sensor networks," in Proc. Workshop on Real-world Wireless Sensor Networks, ser. REALWSN '08. New York, NY, USA: ACM, 2008, pp. 1-5. [Online]. Available: http://doi.acm.org/10.1145/1435473.1435475

[13] H. Liu, H. Darabi, P. Banerjee, and J. Liu, "Survey of wireless indoor positioning techniques and systems," IEEE Trans. Syst., Man, Cybern., Syst., vol. 37, no. 6, pp. 1067-1080, Nov 2007.

[14] K. Chintalapudi, A. Padmanabha Iyer, and V. N. Padmanabhan, "Indoor localization without the pain," in Proc. 16th Annual Int'l Conf. Mobile Computing and Networking, ser. MobiCom '10. New York, NY, USA: ACM, 2010, pp. 173-184. [Online]. Available: http://doi.acm.org/10.1145/1859995.1860016

[15] J. Wan, N. Yu, R. Feng, Y. Wu, and C. Su, "Localization refinement for wireless sensor networks," Comput. Commun., vol. 32, no. 13-14, pp. 1515-1524, Aug. 2009. [Online]. Available: http://dx.doi.org/10.1016/j.comcom.2009.05.011

[16] K. W. K. Lui, W.-K. Ma, H. C. So, and F. K. W. Chan, "Semidefinite programming algorithms for sensor network node localization with uncertainties in anchor positions and/or propagation speed," IEEE Signal Process. Mag., vol. 57, no. 2, pp. 752-763, Feb. 2009.

[17] S. Srirangarajan, A. H. Tewfik, and Z. Q. Luo, "Distributed sensor network localization with inaccurate anchor positions and noisy distance information," in Proc. IEEE ICASSP'07, vol. 3, Honolulu, USA, April 2007, pp. 521-524.

[18] _ - "Distributed sensor network localization using socp relaxation," IEEE Trans. Wireless Commun., vol. 7, no. 12, pp. 4886-4895, Dec. 2008.

[19] Y. Zhou, J. Li, and L. Lamont, "Multilateration localization in the presence of anchor location uncertainties," in Proc. IEEE GLOBECOM2012, Dec 2012, pp. 309-314.

[20] R. Kalman, "A new approach to linear filtering and prediction problems," Trans. ASME - Journal of Basic Engineering, no. 82, pp. 35-45, 1960.
[21] H. T. Friis, "A note on a simple transmission formula," IEEE Trans. Wireless Commun., pp. 254-256, May 1946.

[22] T. Rappaport, Wireless Communications: Principles and Practice, 2nd ed. Upper Saddle River, NJ, USA: Prentice Hall PTR, 2001. 